\title{
DECENTRALIZING PRODUCTS CERTIFICATES USING BLOCKCHAIN TECHNOLOGY
}

\author{
Eduard Daoud and Martin Gaedke \\ Technische Universität Chemnitz, Germany
}

\begin{abstract}
OECD wrote in their report 2016 that up to 5\% of imports are counterfeited goods. The report estimated this damage at EUR 85 billion (OECD/EUIPO, 2016). The presence of counterfeit products on the European market is on the increase, therefore the intervention of inspection bodies and authorities alone is not sufficient, consumers can make their contribution and support this process. 2003 started the EU a program "Rapid Alert System" as an information platform to publish alerts about dangerous products in the market. Over 2000 alerts published by the system yearly. Thus, allowing local authorities to screen their market and take appropriate action should the same product be found (Directorate-General for Justice and Consumers (European Commission), 2018). In this paper, we propose the foundations of a new system for decentralizing product certificate based on blockchain technology which close the gaps of the unilateral published digital certificate. The decentralizing of product certification by using blockchain technology empowers consumers by easily verifying a product certificate, at the same time enable certification bodies to issue and sharing a certificate in tamperproof ways. This could be one of the important steps in the direction to reduce counterfeiting. The goal of this paper is to create a new category of applications in which the end-user identifies counterfeit product certificates and contribute to the fight against product piracy.
\end{abstract}

\section{KEYWORDS}

Blockchain, Decentralization, Information Access, Certification Industry, Counterfeiting

\section{INTRODUCTION AND THE CURRENT PROBLEM}

The problem is that every certificate issuer publishes their certificate on their websites in a way which not easier to retrieve or check the validity of a certificate and interact with the certification marks. One of the possible solutions is to build a central database for all certificates from all certification bodies and inspection agencies as a single point of access but such a platform depends on the certification bodies in the first line which they are in a competitive relationship with each other, this reduces the willingness to implement such solutions much more important is the data inaccuracy and how easy to the fake certificate to go to the market.

Since its launch in 2003, the EU's Rapid Alert System has been providing EU member States with a network and communication tools to publicize counterfeit products. The system stabilizes at regular intervals, about 50 alerts are published each week on the European Commission's website, with slightly more than 2,000 alerts released each year. (Directorate-General for Justice and Consumers (European Commission), 2018).

The number of counterfeits reported products is extremely low in relation to the number of counterfeit products imported into the EU. OECD wrote in their report 2016 that up to 5\% of imports are counterfeited goods. The report estimated this damage at EUR 85 billion (OECD/EUIPO, 2016). Currently, product certificate data are mainly stored separated by the deferent institutions, which hinders the easy information exchange and collaboration between consumer and these institutions. Data security and the competition situation on the market in the first line leads to the fact that the institutions don't share their data to create a single point of access, because the failure to secure the product provider/retailer (as regards the certificate) leads to legal and financial consequences for the institutions.

In the next section, we will introduce the principles of the Blockchain and headline the social value shortly, after introducing the principles of the approach, we examined and substantiated the software stack based on Ethereum Blockchain and other technical components to store and retrieve industry product certificate finally the challenges, conclusion and future works. 


\section{PRINCIPLES OF THE BLOCKCHAIN}

A Blockchain is a distributed append-only store of transactions across computational nodes and structured as a linked list of blocks, each containing a set of transactions (Weber, et al., 2019). Blockchain was introduced as the technology behind Bitcoin (Nakamoto, 2008). The blockchain is generally categorized into two classes (Prusty, 2018):

Table 1. Categories of Blockchain (simplified)

\begin{tabular}{lll}
\hline Blockchain Category & Community & Example \\
\hline Permissioned & proprietary networks & Group of banks \\
Permissionless & opensource networks & Anyone can access and use. \\
\hline
\end{tabular}

A Blockchain is a community topic as a distributed ledger every member of the community could validate any shared information collectively. Any digital assets could be recorded as an information entry. This entry is accessible, permanent, transparent and auditable. These characteristics enable the participants to understand everything at a certain point in time. Information is represented in entries blocks and stored at the end of the chain. The management of the entries and their distribution is the responsibility of blockchain protocol (consensus protocol). There is no central management, monitoring and trust instance in the Blockchain concept. (Grech \& Camilleri, 2017).

To understand blockchain technology more we need to underline the term ledger. Ledgers are a list of sequential transactions with timestamp allowed each participant of the community at any time to determine the owner of an asset (Grech \& Camilleri, 2017). Therefore, Blockchains are a linear sequence of blocks with a timestamp and linked internally cryptographically. There are some characteristics depend on the architecture of the Blockchain and the consensus protocol like immutability, security, validity and transparency.

Some Blockchains are established as a "distributed" network structure and facilitate peer-to-peer transactions across non-hierarchical nodes. Some blockchains, on the other hand, are to be categorized by their consensus protocol for the immutability of their ledgers. In order to understand and classify the impact of the blockchain on society, the topic of social principles of the blockchain should be explored and analyzed in the same way as technical principles. (Grech \& Camilleri, 2017). Following the arguments in (Grech \& Camilleri, 2017) in the educational environment and (Piscini, et al., 2016), in general, an attempt is to be made to propose some blockchain principles that represent social value propositions in the industrial environment like self-sovereignty and identity, trust, transparency and provenance, immutability and disintermediation.

After underlining of the principles for in this paper researched approach, we will not go into the details of the social principles. This is a topic for further leading research work. In order to understand the approach more deeply and after the introduction of the principle of Blockchain we shall lead to possible system architecture and its design to the creation of an industrial product and service certificate.

\section{ARCHITECTURE DESIGN OF THE APPROACHED SYSTEM}

Transactions, smart contract and digital signatures are generally the important records stored in a Blockchain. Transactions have two components digital currency and documentary part of ownership. Smart contracts are computer algorithm stored on a blockchain, describe an agreement between all participants in the Blockchain. Digital signatures are evidence that the statements have been issued by and to the named parties. Before we go deeper into the design of the system for managing industry product certificates, we need first to try to start defining industry certificates and shortly pointing out the demarcation line of the approach.

A certificate, in general, confirms a claim against standards. In the industrial environment, there are three areas for certification: certification of products and services or certification of management systems. A valid certificate can be issued if all financial and technical requirements are met (TÜV SÜD AG, 2019). In certain industries, certification is part of the legal requirements. In general, the certificates increase the credibility of the product or service and affect the expectations and decisions of the consumer. A certificate has essentially the following attributes, these are described in the following table: 
Table 2. Certificate attributes

\begin{tabular}{ll}
\hline Attributes & Brief explanation \\
\hline The claim & Statement/Declaration \\
An issuer & A company/third party that confirms the truth of the statement. \\
Evidence & The description of the test procedures with further clarification about the claim. \\
A recipient & The company/supplier or service provider that has submitted a claim for certification. \\
A certificate & Dataset of different metadata and parameters \\
A certification Mark & As a kind of image or textual representation to demonstrate compliance with certain standards. \\
A signature & A certificate contains a unique code for the product to validate and confirm its identity. \\
\hline
\end{tabular}

After shortly highlighting the topic certificate, the architecture of the system for managing product certificates should cover the involves three processes: issuing, sharing and retrieving. The new approached system should not cover certification in the education sector but only in the industrial area and in the same time it should be able to close the gap of current certificates systems between the consumer and certificate holder.

The current system in which most records are still issued as hybrid certificates (paper and digital) and there is no 'standard format' for certificates. The approached blockchain-based distributed system is based on a public Blockchain and allow a certificate issuer to signs a well-structured certificate and stores its hash within an Ethereum Blockchain transaction. Firstly, we need to go deeper on how is the method to issue a certificate in the Figure:

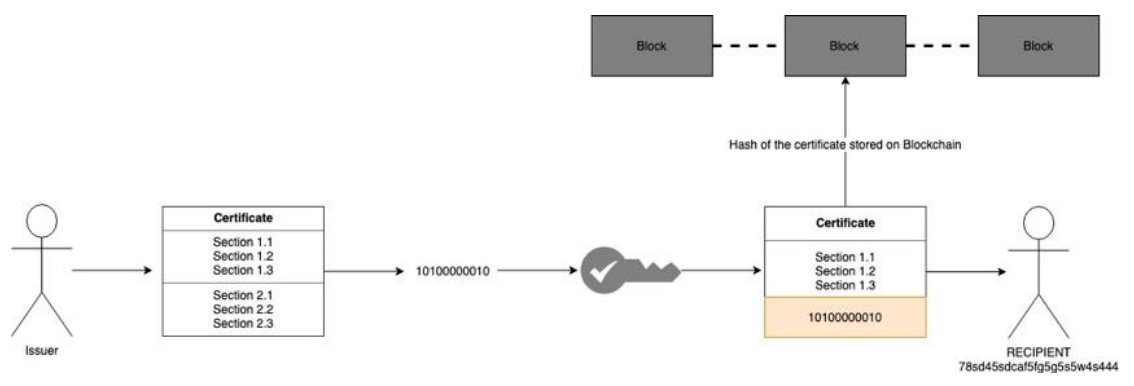

Figure 1. Issuing a certificate on Blockchain

As soon as the certification body creates a certificate in digital form, this file should be issued with some basic information like such as the name of the issuer and recipient and the issue date etc. The issuer shall then cryptographically sign the content of the certificate with a private key to which only the issuer has privileged access. The issuer of the certificate stores his signature in the certificate dataset. The cryptographic hashing the credentials information to allowed users to check the contents of the certificates against manipulation. In the end, the issuer uses his private key again to write the record in the blockchain.

The issuer makes the certificate available to the recipient (sharing), e.g. directly for accessing on his website. The important point in publishing and sharing the certificate is to ensure that every other internet user can access the certificate. The recipient can mention his digital certificates on his products or make them available on his website. Now, all basic requirements are fulfilled so that the end-consumer can simply check an issued certificate (retrieving). The system uses a permissionless (public) Blockchain for issuing certificates, this means that anyone connected to the network could validate and verify the certificate information with help of the smart contract address.

After introducing the three main processes of the approaches system: Issuing, sharing and retrieving of the certificate, the next figure addresses the high-level system component overview:

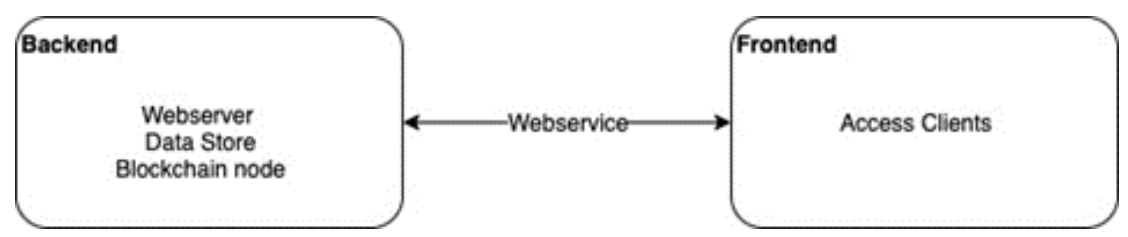

Figure 2. High level system overview 
The high-level System overview provides an overview of the components which spat into frontend and backend as a main component. The frontend consists of different access clients. The backend based on a web server, a local Datastore and also runs a full Blockchain node (Ethereum). A more detailed view of the server architecture and using smart contract is provided in the next section.

\section{SOFTWARE STACK BASED ON ETHEREUM BLOCKCHAIN}

Ethereum provides a universal Blockchain combined with a Turing full programming language to enable the development and implementation of applications that make use of the Blockchain (Vitalik, 2014). Moreover, Hyperledger is also open-source technology which allows developers to set up their private network to advance cross-industry blockchain technologies (Grech \& Camilleri, 2017).

We choose Ethereum Blockchain technology based on the following criteria. Transparent: Using Hyperledger is not possible, because the approached system should be a public blockchain network. Smart Contract: Allow using smart contracts to define some runnable code for negotiation and facilitation. To demonstrate the approach from the technical side we need to go deeper from the high-level overview to a software stack description of the two main components. The next figure explains the two main components:

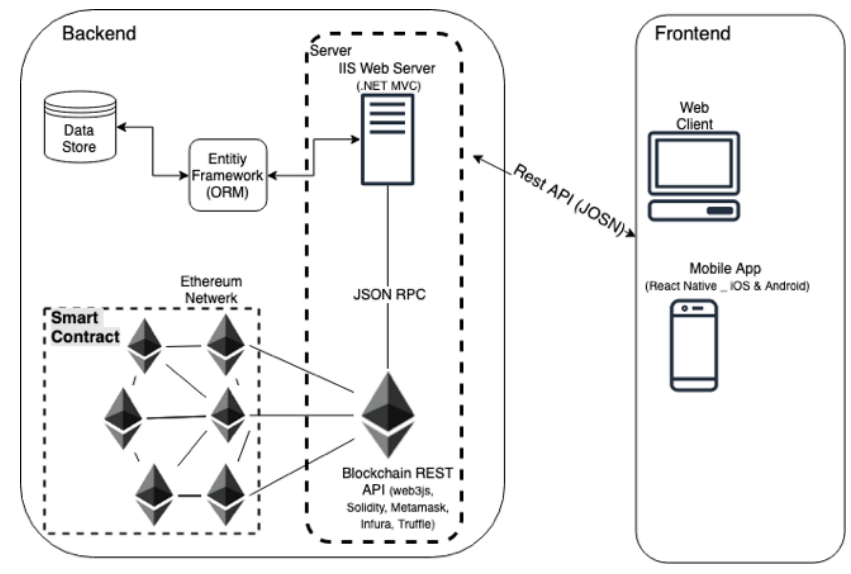

Figure 3. Technical architecture

The frontend is a public web application act as access clients (.NET MVC) communicate with a web server runs on IIS in the backend. The backend has also an internal JavaScript runtime environment (NodeJS). The two main components communicate with each other via the REST API (JSON) to handle user requests and interact with the Ethereum network. The web application framework is ASP.NET MVC an open-source framework, which implements the model-view-controller pattern. The webserver provides RESTful service endpoint. The most crucial part of the approach is the internal part (Backend) that interacts with the Ethereum network. The public web application handles user requests and interacts with NodeJS within IIS Web server to process these data that related to Ethereum network, such as: create a new smart contract, deploy it to Ethereum node, query data or search information.

Both parts are also connected to our internal management relational databases to make sure that these data have to be consistent. To interact with a smart contract, we use Nodejs to build our interaction part with the Blockchain network and Metamask to create and manage Blockchain wallet to connect to the network. To sign a smart contract, the system needs a tool like Truffle to sign the transaction. Importantly, data such as certificate information are not stored directly in the block of the Ethereum. Only the root node hashes of the transaction, state and receipt are stored directly in the blockchain to gain more experience and test how to store and retrieve certificate information from Ethereum Blockchain we started a new research process handle these two points separately.

In the following section, we discuss the challenges of our approach bevor we headline the summary and an outlook on our work. 


\section{CHALLENGES}

From the analysis of the selected literature, a series of insights can be derived concerning the challenges of the approached blockchain-based distributed systems and its usability across a wide area of industry certification. However, like any other emerging technology, issues and challenges arise. One of the challenges in decentralizing of product certificates using blockchain technology is the integrity of data, followed by blockchain confidentiality and the willingness to share information.

Many companies include the data collected from the customer in their assessment of the value-added process to create new products and or services. Therefore, protecting privacy should be placed when considering how such records could be integrated into a Blockchain. Confidentiality involves the protection of data from unauthorized disclosure, either by direct retrieval or by indirect logical inference. Among others, issues such as third-party confidentiality violations are being scrutinized.

All changes in the Blockchain are to be acknowledged and stored audit-ably, that could be the best way to ensure the integrity of the data. This preserves the accuracy, consistency, and validity of the data throughout its lifecycle. Searchability, recoverability, traceability and connectivity of certificate data are some important properties provided by ensuring the integrity of data. The integrity of data should be comprehensively researched and some projects as Enigma in MIT (Zyskind, et al., 2015) could make a valuable contribution.

The willingness to share information from testing and inspections agencies is one of the no-technical challenges. By doing a small experimental project with real users and with one of the certification bodies could be a good start to demonstrate the long-term effects of the approach. In general, there are several of challenges and limitations of the blockchain technology these are comprehensively addressed in this paper (Casino, et al., 2019, pp. 55-81) like suitability of Blockchain, latency and scalability and blockchain adoption and interoperability. After highlighting the challenges, we headline a summary and an outlook on our work.

\section{CONCLUSION AND FUTURE WORKS}

This paper presents a new approach as platform architecture to decentralizing products certificates using blockchain technology. We evaluate the solution by using a Bitcoin Blockchain as a proof of concept focused on feasibility in this paper (Daoud, 2019) and found a lot of limitations.

The blockchain-based distributed approach used in core standard web technologies and Ethereum network. The conclusions we can define from the new approach are that the implementation of the system should be deeper researched, from the point of view of completely open blockchain or hybrid. The main focus is on which implementations have a positive impact on anti-counterfeiting of products and the adoption of blockchain-based certificates depends on how the consumer can easily and simply interact with the system.

In future work, we plan to explore and research more about using machine learning to classify certificate marks and logos of certification bodies to unleash the potential of blockchain-based certificate retrieving possibilities shortly we are intending to provide a better way of giving information, by scanning the products itself and using AI-based Image recognition. This would combine two state-of-the-art technologies, machine learning and blockchain, in one application. That will bring great convenience and a better experience for users.

However, we trust that the decentralizing of product certification by using blockchain technology will change the role and empower the consumer to drive the market for more transparency.

\section{REFERENCES}

Casino, F., Dasaklis, T. K. \& Patsakis, C., 2019. A systematic literature review of blockchain-based applications. Telematics and Informatics, pp. 55-81.

Daoud, E., 2019. Decentralizing of Transparency: Using Blockchain to Reduce Counterfeiting. Utrecht, the Netherlands, IADIS Press, p. 8.

Directorate-General for Justice and Consumers (European Commission), 2018. Results of the EU rapid alert system for dangerous non-food products, Brussels: European Commission.

Elmasri, R. \& Navathe, S. . B., 2015. Fundamentals of Database Systems. s.1.:Pearson. 
Ethereum Foundation (Stiftung Ethereum), 2018. Ethereum Project. [Online] Available at: https://www.ethereum.org/ [Accessed 1908 2019].

Gideon, G., 2017. Blockchains vs Centalized databases. [Online] Available at: https://www.multichain.com/blog/2016/03/blockchains-vs-centralized-databases/ [Accessed 1901 2019].

Grech, A. \& Camilleri, A. F., 2017. Blockchain in Education, European Union: European Union.

Nakamoto, S., 2008. Bitcoin: A Peer-to-Peer Electronic Cash System. [Online] Available at: https://bitcoin.org/bitcoin.pdf [Accessed 1807 2019].

OECD/EUIPO, 2016. Trade in Counterfeit and Pirated Goods: Mapping the Economic Impact. Paris: OECD Publishing.

Peters, G. C. A. \&. P. E. J. B. R., 2016. Opening discussion on banking sector risk exposures and vulnerabilities from Virtual currencies. Journal of Banking Regulation, Issue 4, p. 239-272.

Peters, G. W. \& Panayi, E., 2015. Understanding modern banking ledgers through blockchain technologies. Computers and Society (cs. CY); Cryptography and Security (cs.CR), pp. 5-6.

Piscini, E., Guastella, J., Alex, R. \& Nassim, T., 2016. Deloitte University Press.. [Online] Available at: https://www2.deloitte.com/content/dam/Deloitte/uk/Documents/technology/deloitte-uk-tech-trends2016-blockchain.pdf [Accessed 2809 2019].

Prusty, N., 2018. Blockchain for Enterprise: : Build scalable blockchain applications with privacy, interoperability, and permissioned features. Birgmingham: s.n.

Redmond , E. \& Wilson, J. R., 2012. Seven Databases in Seven Weeks: A Guide to Modern Databases and the NoSQL Movement. s.l.:North.

Remeur, C., 2017. Market surveillance and product safety, Bruessel: European Parliament.

Sirpa, P., Françoise, G. \& Bart, S., n.d. Member States should stop turning a blind eye to product safety, s.l.: The Parliament Magazine.

TÜV SÜD AG, n.d. Certificate Explorer. [Online] Available at: https://www.tuev-sued.de/product-testing/certificates [Accessed 0609 2019].

TÜV SÜD AG, n.d. Misuse of quality marks. [Online] Available at: https://www.tuev-sued.de/producttesting/_services/markets_and_competition/quality_and_test_marks/abuse_of_quality_marks [Accessed 1208 2019].

Tabora, V., 2018. Hacker Noon. [Online] Available at: https://hackernoon.com/databases-and-blockchains-the-differenceis-in-their-purpose-and-design-56ba6335778b [Accessed 1901 2019].

The Netherlands Court of Audit, 2017. Products sold on the European market: unravelling the system of CE marking, Netherlands: House of Representatives.

Vitalik, B., 2014. A next-generation smart contract and decentralized application platform. Ethereum White Paper, Volume 3, p. 37.

Vukolić, M., 2016. The quest for scalable blockchain fabric: Proof-of-work vs. BFT replication. Zurich, Springer Verlag.

Weber, I. et al., 2019. A Platform Architecture for Multi-Tenant Blockchain-Based Systems. IEEE International Conference on Software Architecture (ICSA2019), p. 10.

Zhao, J., Fan, S. \&. Y. \& J. , F. I., 2016. Overview of business innovations and research opportunities in blockchain and introduction to the special issue. Financial Innovation, 2(1), p. 28.

Zyskind, G., Nathan, O. \& Pentland, A., 2015. Enigma: Decentralized Computation Platform with Guaranteed Privacy. Cryptography and Security. 